\title{
Reflections on reflections of Dieter Bothe on the 'litmus test' for mathematical models of dynamic wetting
}

\author{
Eur. Phys. J. Special Topics 229, 1979 (2020), \\ https://doi.org/ 10.1140/epjst/e2020-000149-6
}

Yulii D. Shikhmurzaev ${ }^{\mathrm{a}}$

School of Mathematics, University of Birmingham, Birmingham B15 2TT, United Kingdom

Received 6 July 2020 / Accepted 6 July 2020

Published online 14 September 2020

\begin{abstract}
The comments of Dieter Bothe on the 'litmus test' proposed in [Y.D. Shikhmurzaev, Eur. Phys. J. Special Topics 229, 1945 (2020)] as a simple way of assessing adequacy of mathematical models of dynamic wetting are examined and shown to be erroneous with regards to two of the flow configurations described in the 'litmus test' and missing for the remaining two. Therefore, the conclusions of Bothe's paper [D. Bothe, Eur. Phys. J. Special Topics 229, 1979 (2020)] should be regarded as completely unfounded.
\end{abstract}

Before addressing Bothe's comments [1], it seems reasonable to put them and the 'litmus test' described in [2] as well as the whole debate in a broader context.

For over 50 years, the mathematical modelling of dynamic wetting in the framework of continuum mechanics has been dominated by an approach where the dynamic contact angle $\theta_{d}$ formed by a free surface with a solid substrate is an input into the model, a prescribed function of the speed $U$ at which the three-phase contact line moves across the substrate:

$$
\theta_{d}=f\left(U, \theta_{s}, k_{1}, k_{2}, \ldots\right)
$$

where $\theta_{s}$ is the static (equilibrium) contact angle and $k_{1}, k_{2}$, etc. are material constants characterizing the contacting media.

This approach, initially undisputable, started crumbling as experiments, first on 'hydrodynamic assist of dynamic wetting' [3-5] and then on 'spontaneous' versus 'forced' wetting [6,7], began to discover, with increasing certainty, that a single function $f$ in (1) is unable to describe the data. Nevertheless, the supporters of/belivers in this approach, which they somewhat prematurely termed 'standard', could still hold their ground by questioning the accuracy of the neasurements and/or by attributing the discrepancy to physical factors additional to dynamic wetting not accounted for in the 'standard' models.

a e-mail: Y.D.Shikhmurzaev@bham.ac.uk 
What the field needed was an argument as undeniable as Galileo's thought experiment which answered the then unsettling question whether bodies of different mass are falling down with the same acceleration. As some of us remember, Galileo tried to answer this question by dropping metal balls of different mass from the appropriately leaning Tower of Pisa but the results were inconclusive - just like the dynamic wetting experiments are today - and his successors could have been dropping objects from available heights to the present day should he not come up with a simple argument involving two balls of different mass first taken separately and then joined to form a single object. This simple idea drew a line under the whole debate, so that nowadays, when we watch a video posted on YouTube where a feather and a metal ball are falling down side by side in NASA's high-vacuum chamber, we, thanks to Galileo's thought experiment, see this as an illustration of the already established fact rather than an element of ongoing research. The field of dynamic wetting needed a similar kind of argument which would help the defenders of the 'standard' approach revise it or at least stop calling it 'standard'.

In [2], a Galileo-style family of experiments has been proposed to show the fallacy of the modelling approach based on (1). These experiments are formulated in the form of a 'litmus test' and illustrated with four flow configurations. Each of these flow configurations and the associated diagram in the angle-versus-speed plane completely undermines equation (1) as a key element in the 'standard' approach.

The reason for giving four flow configurations and not just one was as follows. A staunch believer in (1) can always come up with some ad hoc arguments, convincing, albeit mostly to him/herself, that would allow him/her to dispute one flow configuration. However, once he/she moves on to the next flow configuration, he/she will discover that these arguments do not work and a new set of ad hoc arguments has to be invented. Should he/she move on to the third configuration, it will become apparent that neither set of ad hoc arguments invented for the previous cases works and something even more elaborate has to be brought in. Eventually, it might occur even to the staunchest believer in equation (1) that it is this article of faith that is at fault, not the experiments that invalidate it. Thus, the proposed flow configurations collectively would act as a self-education toolkit allowing one to look at the dynamic wetting process in an undogmatic way and deeper understand it. Once this is achieved, the now converted former believer might even go back to and find flaws in his/her own ad hoc arguments invented earlier, thus sparing me, as the originator of the 'litmus test', from entering a debate to rebut them. Admittedly, this optimistic scenario relies on the reader of [2] being prepared to examine all arguments without jumping to conclusions and, if necessary, even play devil's (i.e. my) advocate, which seemed to be indeed the case in Galileo's times even with the Inquisition around.

The 'reflections' of Dieter Bothe [1] followed the above scenario but only halfway as he considered two flow configuration out of four and nevertheless somehow felt it appropriate to draw conclusions about the 'litmus test' as a whole. So, I have to step in and, once this has to be done, use this opportunity to clarify a few points.

Bothe begins with the first flow configuration, namely a drop spreading over a flat solid substrate. He then spends more than a half of his entire paper arguing that the drop cannot spread if it contacts the substrate at a single point. Even 'homeomorphism' is brought in to strengthen the argument. However, should Bothe's reflections on [2] be as careful as promised in the abstract of his paper, he would have noticed that he is breaking into an open door as all this is already said in the text he reviews. Indeed, in [2] one can read:

"Initially, the contact angle is $180^{\circ}$, which is simply a mathematical fact as there are two smooth surfaces touching. Once pressed against the substrate, e.g. by gravity, the drop will form an infinitesimally small area of 
contact with the solid and this [!] will trigger its spreading over the substrate. Thus, the motion begins from rest $(U=0)$ and from the contact angle of $180^{\circ}$ (or infinitesimally close to it). This starting point is shown as (a) in the contact angle-versus-speed plane [...]."

As we can see, it has been emphasized that it is only after the drop touching the substrate at a point is pressed against it (infinitesimally slowly to avoid an impactlike effect) to form an area of contact that it starts to spread. How could this point have been made any clearer?

It is not clear whether the author of [1] failed to read/understand the piece quoted above or chose to ignore it and argue as if there is a conflict between what his homeomorphism is telling him and what is already said in [2] in plain English. The fact remains: (a) the reader has been misled as to what [2] actually states regarding the first flow configuration and (b) more than a half of [1] is wasted discovering what to (almost) everyone working in dynamic wetting is simply commonplace. Moreover, Bothe even brings in an experimental picture, admitting that it does not actually answer any question. Why is it brought in then?

The rest of Bothe's argument revolves around the scaling law

$$
r \sim t^{1 / 2}
$$

where $r$ is the radius of the drop's area of contact with the substrate and $t$ is the time; see (15) in [1]. This scaling law implies that at the onset of the process $(t=0)$ the contact-line speed is infinite, and the author discusses at length how this should be understood. However, (2) is, so to speak, an 'abbreviated' form of the scaling law in question, the scaling law with no scaling parameters involved, whilst if we look at it in its full form, it becomes clear immediately that the reader has been misled once again. Indeed, without an abbreviation the scaling law (2) has the form

$$
r \sim\left(R U_{\text {impact }}\right)^{1 / 2} t^{1 / 2}
$$

where $R$ is the radius of curvature of the free surface at the point of the initial contact and $U_{\text {impact }}$ is the speed at which the drop impacts onto the solid substrate. In other words, this scaling law corresponds to the so-called 'kinematic' or 'geometric' phase of wetting following the drop impacting a substrate at a finite speed $U_{\text {impact }}$. Looking back at the piece from [2] quote above, one can see that the impact-driven flow examined by Bothe has nothing to do with the flow considered in the 'litmus test', where the whole idea was to initiate the dynamic wetting process from rest by pressing - infinitesimally slowly! — the drop against the substrate to create an area of contact and thus trigger the process of its spontaneous spreading. In short, the 'litmus test' is about 'spontaneous' spreading whilst Bothe is looking at the 'forced' spreading (or, more accurately, the even more 'violent' stage of kinematic/geometric wetting preceding it). Thus, the reader again appears to have been misled as the scaling law (2), i.e. (3), is irrelevant to the flow considered in [2] and its discussion in [1] has nothing to do with the 'litmus test'.

After spending almost all of his 'reflections' on the first flow configuration of the 'litmus test', Bothe comes to the second one only to find that his preceding comments have been anticipated and none of them now applies, so that, as in the scenario outlined above, a new set of ad hoc arguments has to be invented. Indeed, for a drop deposited onto and then spreading over a suitably profiled substrate (Fig. 1 here reproducing Fig. 4 of [2]), the finite area of contact is present from the start and no irrelevant impact-based scaling law that would help to argue in favour of an infinite starting velocity is available. Thus, one is left with no option but to consider the drop 


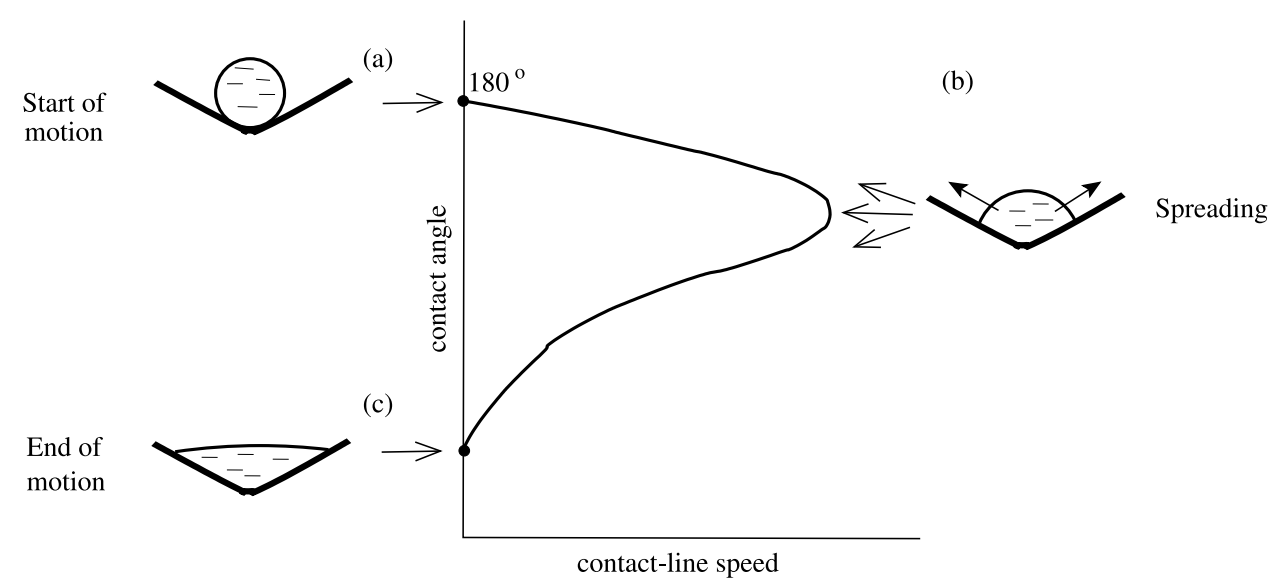

Fig. 1. The second flow configuration - Figure 4 of [2]. A drop touches, slowly, without any impact, a profiled substrate across a finite area and the motion begins, as all motions do, from rest. The corresponding flow diagram in the contact angle-versus-speed plane completely undermines (1) as an element of any realistic mathematical model of dynamics wetting.

spreading from a finite base and from rest. Well, not quite as now the author's new line of argument goes as follows:

"This gedankenexperiment would require that the gas film drains without any deformation of the interface [...]".

It does not require too deep 'gedanken' to realize that there will be no deformation of the interface by the gas film if (a) there is no gas, e.g. when a drop of a low-volatility liquid is placed on a substrate in a vacuum chamber, and/or (b) the drop is brought in contact with the solid infinitesimally slowly — a "gedankenexperiment" is a perfect instrument for doing this. This is not to mention that the substrate's profile could happen to be exactly the one matching the shape of a deformed drop. As we can see, now Bothe has to bring in factors additional to dynamic wetting, not inseparable from it.

Let us follow Bothe's argument further:

"[...] and that the initial wetting simultaneously happens on the full spherical cap region, while the onset of wetting requires a nucleation-like process to trigger the topological change".

Now we can witness a peculiar logical twist. In dealing with the first flow configuration, the author considers how an almost flat free surface approaches a flat solid boundary. He even draws a picture (Fig. 3 of [1]) illustrating this process in minute detail. However, when he is offered a substrate with the profile matching the shape of the approaching drop, he refuses to consider it in the same way and, instead of returning to his own Figure 3 and suitably modifying it to see what happens, tries to argue his way out of what for the 'standard' approach is a dead end by inventing a "nucleation-like process" so far unheard of. Does Figure 3 of [1] with a curved substrate rather than a flat one suddenly become invalid?

In this connection, perhaps, it is worth noting that the so-called 'contact problems' where an initial contact between bodies, solid or liquid, takes place across a finite area routinely appear in all branches of continuum mechanics, in fluid mechanics as well as in elasticity and plasticity. Should one revise all of them by introducing "nucleationlike processes" or is it only dynamic wetting that is an unfortunate exception? 
This "nucleation-like process" merits a separate comment. It is in boiling or solidification that one typically comes across such a process but there is a difference of principle between dynamic wetting and, say, boiling: dynamic wetting is a process by which a liquid-solid interface is produced from an already existing liquid-gas one whilst in boiling a new bulk phase together with a new liquid-gas interface appears. A "nucleation-like process", like in boiling or solidification, is required as an external trigger (hence nuclei as external objects) in a metastable system to produce a new phase whilst in our case (a) the liquid-gas interface is already present and has only to evolve into the liquid-solid one as the drop touches the solid, and (b) the whole process of the droplet deposition onto the substrate, which triggers the liquidgas interface evolution into the liquid-solid interface, is driven externally, i.e. by the one who deposits the drop onto the solid. Therefore, there is absolutely no need in fantasies about any "nucleation-like process' for the process to be described in the framework of continuum mechanics.

Those who prefer to think in down-to-earth terms can see it this way: a flat solid, e.g., physically, cleft mica, which is smooth down to the molecular scale, touching a flat surface of a liquid (also smooth down to the molecular scale) would create a fresh liquid-solid interface (out of equilibrium at first and then equilibrating) across a finite area, at least in a 'gedankenexperiment' where the two surfaces can be strictly parallel, without any "nucleation-like process". Unless one comes from the continuum down to the molecular-level description, where, incidentally, the very term 'wetting' no longer makes sense (except for Bothe who writes about "the fluid molecules which actually are in contact to the substrate [thus wetting it]").

The last argument in [1] reads:

"Moreover, in this thought experiment, the surface energy of the droplet surface contacting at once the spherical cap would be assumed to just disappear without any effect".

Instead of making this assumption, one should remember that an 'interface' is not something suspended in a vacuum with the energy nowhere to go; it is between the bulk phases and, if it finds itself out of equilibrium, e.g. with an excess of energy, it will release energy into the bulk phases. An interface can even disappear altogether, e.g. in the process of coalescence where two fluid bodies are brought in contact such that parts of their free surfaces become trapped between them $[8,9]$, but even then the energy will not "just disappear without any effect": it will go into the internal energy of the bulk phase raising its temperature albeit by a very small amount as the energy associated with the interface is small compared with that of the bulk.

In summary: the energy associated with an interface is determined by what bulk phases have this interface between them. Once the former liquid-gas interface (the part by which the drop contacts the solid) finds itself between the liquid and the solid phases, the process of its equilibration, i.e. relaxation to its new environment, will start, and the interface will exchange mass, momentum and energy with the bulk phases until it becomes an equilibrium liquid-solid interface. Incidentally, this is what the entire monograph [10] is all about. In short, one should remember that the liquid-solid interface has its own energy. This fact has been extensively dealt with by Gibbs [11] and many researchers after him, notably I. Prigogine and P. Mazur, so that one would have expected this fact to be known. It looks though that Aristotle was right after all when he remarked that "what 'is known' is known to a few".

Now, let us move on to the third and fourth flow configurations in [2], namely (iii) a meniscus rising in a capillary tube after the latter touches a horizontal free surface and (iv) the meniscus moving and equilibrating after a wedge (or a cone) dips into a pool of liquid. For these flows, one can neither bring in any scaling laws, even irrelevant ones, as there are no scaling parameters to use in them nor can one apply the arguments quoted above as neither the presence/absence of the ambient gas nor 
any musings about imaginary "nucleation-like processes" would make any difference. And, as we remember, each of the flow configurations given in the 'litmus test' has a flow diagram similar to that shown in Figure 1 and hence completely undermines the 'standard' approach based on (1). So, what counterarguments against (iii) and (iv) are to be expected? We have no answer as the "careful consideration" promised in the abstract of [1] terminates without considering, carefully or otherwise, these flows.

Thus, we have to conclude that Dieter Bothe's reflections [1] on the 'litmus test' described in [2] failed even to shake, leave alone disprove, a single point regarding the first two flow configurations in [2] and did not even touch upon the remaining two. Therefore, the conclusions made by the author of [1] regarding the 'litmus test' have to be regarded as completely unfounded.

On a personal note, as much as I am in favour of debate as an enjoyable thoughtprovoking intellectual exercise and even have a track-record of provoking one [12-21] by publishing a polemic piece [22], albeit after an invitation and encouragement from M.G. Velarde, editor of the special issue on dynamic wetting, it is disconcerting to notice that the debate does not seem to be moving towards deeper issues than the agenda of the 1970s, e.g. kinetic effects in dynamic wetting [23] or the new challenges outlined in [2]. Each of them would make one rethink 'classical' dynamic wetting through the prism of a broader physical context into which dynamic wetting would now have to be embedded, and ad hoc constructions would simply stand in the way and have to be re-examined and removed. This would also put an emphasis on consistency in mathematical modelling throughout, put it at the centre of the research agenda, and hence require investigators to be equipped appropriately in this regard. These somewhat sobering observations make a discussion about the state of and schooling in mathematical modelling started in the concluding section of [2] and the lessons to be drawn from the experience of the past 50 years both relevant and timely.

The support of the Engineering and Physical Sciences Research Council (U.K.) under Grant $\mathrm{EP} / \mathrm{P} 031684 / 1$ is acknowledged.

Open Access This is an open access article distributed under the terms of the Creative Commons Attribution License (http://creativecommons.org/licenses/by/4.0), which permits unrestricted use, distribution, and reproduction in any medium, provided the original work is properly cited.

Publisher's Note The EPJ Publishers remain neutral with regard to jurisdictional claims in published maps and institutional affiliations.

\section{References}

1. D. Bothe, Eur. Phys. J. Special Topics 229, 1979 (2020)

2. Y.D. Shikhmurzaev, Eur. Phys. J. Special Topics 229, 1945 (2020)

3. T.D. Blake, A. Clarke, K.J. Ruschak, AIChE J. 40, 229 (1994)

4. T.D. Blake, M. Bracke, Y.D. Shikhmurzaev, Phys. Fluids 11, 1995 (1999)

5. A. Clarke, E. Stattersfield, Phys. Fluids 18, 048109 (2006)

6. M.J. Davis, S.H. Davis, C. R. Phys. 14, 629 (2013)

7. A.M. Karim, S.H. Davis, H.P. Kavehpour, Langmuir 32, 10153 (2016)

8. J.E. Sprittles, Y.D. Shikhmurzaev, Phys. Fluids 24, 122105 (2012)

9. J.E. Sprittles, Y.D. Shikhmurzaev, J. Fluid Mech. 751, 480 (2014) 
10. Y.D. Shikhmurzaev, Capillary flows with forming interfaces (Chapman, Hall/CRC, Boca Raton, London, New York, 2007)

11. J.W. Gibbs, Collected works of J. Willard Gibbs (Longmans, Green \& Co., New York, 1928), Vol. 1

12. U. Thiele, Eur. Phys. J. Special Topics 197, 67 (2011)

13. Y.D. Shikhmurzaev, Eur. Phys. J. Special Topics 197, 221 (2011)

14. L.M. Pismen, Eur. Phys. J. Special Topics 197, 63 (2011)

15. Y.D. Shikhmurzaev, Eur. Phys. J. Special Topics 197, 75 (2011)

16. Y. Pomeau, Eur. Phys. J. Special Topics 197, 81 (2011)

17. Y.D. Shikhmurzaev, Eur. Phys. J. Special Topics 197, 85 (2011)

18. J.R. Henderson, Eur. Phys. J. Special Topics 197, 61 (2011)

19. Y.D. Shikhmurzaev, Eur. Phys. J. Special Topics 197, 125 (2011)

20. T.D. Blake, Eur. Phys. J. Special Topics 197, 343 (2011)

21. Y.D. Shikhmurzaev, Eur. Phys. J. Special Topics 197, 73 (2011)

22. Y.D. Shikhmurzaev, Eur. Phys. J. Special Topics 197, 47 (2011)

23. J.E. Sprittles, Phys. Rev. Lett. 118, 114502 (2017) 